\title{
Cuing efficiency in a Stroop-like task with visual half-field presentation
}

\author{
MIRJAM EGLIN \\ Swiss Federal Institute of Technology, Zurich, Switzerland \\ and University of California, Berkeley, California \\ and \\ ANDRES HUNTER \\ Swiss Federal Institute of Technology, Zurich, Switzerland
}

\begin{abstract}
Stroop-like stimuli were presented to either the left or the right visual half-field. Subjects responded to the identity of the words ABOVE and BELOW (the target dimension), which appeared above or below a reference point (the cuing dimension). Automatic Stroop-like effects were assessed as the difference in reaction times between congruent trials (e.g., ABOVE above the reference point) and incongruent trials (e.g., ABOve below the reference point) when both trial types were equally frequent. In blocks in which most trials were of one type (e.g., $80 \%$ congruent trials), controlled Stroop-like effects could be assessed. Automatic Stroop-like effects remained unchanged under different task manipulations. In contrast, controlled Stroop-like effects were reduced by lowering cue-response compatibility and by increasing the response alternatives from two to four. Thus, similar to other cuing effects, controlled Stroop-like effects are susceptible to manipulations that affect the response-decision stage and appear to involve response-selection processes. The resources supporting these response-selection decisions were not hemisphere-specific, and were sufficiently nonspecific that interference from a memory-load task was found. When resources were scarce, a consistent bias to attend to stimuli presented or responded to on the right was evident.
\end{abstract}

Several recent research efforts have been aimed at discovering the mechanisms involved in encoding and utilizing precue-target-response contingencies. Typically, precues contain partial information about a subset of targets and precede a target on each trial. Converging evidence suggests that precues facilitate response-decision processes (e.g., see Hendrikx, 1986; Proctor \& Reeve, 1985, 1986; Reeve \& Proctor, 1984, 1985). For example, it has been shown that cue-response $(\mathrm{C}-\mathrm{R})$ and stimulus-response (S-R) compatibility relations, which are thought to affect response-decision processes (Hendrikx, 1986; Reeve \& Proctor, 1984, 1985), interact with precuing efficiency in a variety of paradigms. Thus, precues are thought to facilitate response-decision processes (Hendrikx, 1986; Proctor \& Reeve, 1985, 1986; Reeve \& Proctor, 1984; 1985; but see also Miller, 1982, $1983,1985,1987$ ).

In the present study, it will be shown that C-R and S-R compatibility, as well as other task variables that affect

This research was supported by Research Grant $0.330 .086 .52 / 1$ from the Swiss Federal Institute of Technology and a Swiss National Science Foundation postdoctoral fellowship to the first author. We are grateful to Dani Brandeis, Robert W. Proctor, Gordon D. Logan, and two anonymous reviewers for many helpful comments on previous drafts of the manuscript, and to Curtis Hardyck for providing the laboratory facilities to conduct Experiments 3 and 4. Correspondence may be addressed to Mirjam Eglin, Witikonerstr. 454, CH-8053 Zurich, Switzerland. response decisions, interact with Stroop-like effects. This interaction is taken as evidence that Stroop-like effects also involve response-selection decisions. In Stroop-like tasks, single two-dimensional stimuli are presented. Subjects respond to one dimension (the target dimension) and ignore the other (the cuing dimension). Stroop-like effects occur because there are few response alternatives (La Heij, Van der Heijden, \& Schreuder, 1985) and subjects use the cuing dimension to build up evidence in favor of one response over others (Dunbar \& MacLeod, 1984; Logan \& Zbrodoff, 1979).

For example, the words ABOVE and BELOW may be presented above or below a reference point. Subjects are instructed to respond to the identity of the words, but not to their position. Stroop-like effects are assessed as the difference in reaction times (RTs) between congruent trials (e.g., ABOvE appearing above the reference point) and incongruent trials (e.g., ABOVE appearing below the reference point). When each word appears equally often above and below the reference point, the cuing dimension is not systematically related to the target dimension. In this case, processing of the cuing dimension is considered to be automatic (Posner \& Snyder,1975; Shiffrin \& Schneider, 1977). With automatic processing of the cuing dimension, responses to the target dimension are faster if the two dimensions are congruent than if they are incongruent.

Processing of the cuing dimension is considered to be attentionally controlled, on the other hand, if a system- 
atic cuing relation to the target exists. For example, if $80 \%$ of trials are congruent and $20 \%$ are incongruent, the upper location predicts the response "above" on most trials. In contrast, if most trials are incongruent, the upper location is associated with the response "below." The fact that the unreported dimension reliably predicts one response alternative incites subjects to pay attention to the unreported dimension and results in controlled cuing effects.

In a controlled cuing situation, facilitation obtains for the more frequent type of trial and interference results for the less frequent type (Logan, 1980; Logan \& Zbrodoff, 1979). In other words, responses are faster to congruent trials than to incongruent trials if most trials are congruent, whereas responses may be faster to incongruent than to congruent trials when incongruent trials are more frequent, reversing the usual Stroop-like effect (Logan, 1980; Logan \& Zbrodoff, 1979).

This reversal of the typical Stroop-like effect when incongruent trials are frequent is usually not complete. This may be due to a type of compatibility effect between cue and response (C-R compatibility). It is less natural to associate the upper position with the response "below" and the lower position with the response "above" when incongruent trials are frequent than to associate the upper position with the response "above" and the lower position with the response "below" when congruent trials are frequent.

Other evidence that C-R compatibility contributes to Stroop-like effects comes from tasks in which the processing mode of the cue (e.g., linguistic/spatial) and the response mode (e.g., verbal/manual) are varied. It has been shown that compatible $\mathrm{C}-\mathrm{R}$ relations (e.g., linguistic/verbal) produce larger Stroop-like effects than do incompatible C-R relations (McClain, 1983; Virzi \& Egeth, 1985).

The same basic task with visual half-field (VF) presentation was used in all experiments of the present study. The first experiment replicated the automatic and controlled Stroop-like effects demonstrated by Logan and associates (Logan, 1980; Logan \& Zbrodoff, 1979). In addition, the efficiency of controlled cuing was compared between blocks that differed in their $\mathrm{C}-\mathrm{R}$ compatibility. In the second experiment, we tested whether a memory load could compete for the resources involved in response decisions and could reduce the controlled Stroop-like effects. In the third experiment, response decisions were made more complex by increasing the number of response alternatives from two to four. In addition, in Experiment 3 , we examined whether response-selection decisions could be supported by independent hemispheric systems. Finally, in Experiment 4, the influence of spatial compatibility on controlled cuing effects was examined. Each of these experiments will be introduced separately.

\section{EXPERIMENT 1 TWO RESPONSE ALTERNATIVES}

The first experiment was run to replicate the controlled Stroop-like effects reported by Logan and associates (Lo- gan, 1980; Logan \& Zbrodoff, 1979) in a task with VF presentation. Subjects responded to the identity of the words ABOVE and BELOW, which were presented either above or below an "asterisk" on the computer screen. The word-asterisk configuration was shown on either the left or the right side of the screen.

VF presentation allowed the proportion of congruent and incongruent trial types to vary independently for each VF. Consequently, the association of a cue with a response varied independently in each VF as well. The proportion of congruent trials was the same in both VFs in the homogeneous blocks. In these blocks, the upper stimulus location in each VF was associated with one response alternative, the lower location with the other. In other words, a single stimulus feature was associated with one response alternative (e.g., the upper location with the response "above"). This is considered a situation with high C-R compatibility, in the sense that a salient stimulus feature is associated with a salient response feature (Proctor \& Reeve, 1986). In the mixed blocks, on the other hand, the proportion of congruent trials was high in one VF, but low in the other. Thus, the upper position might have been associated with the response "above" in one VF, but with the response "below" in the other. Therefore, no single stimulus feature could be mapped consistently onto a response feature, resulting in reduced C-R compatibility. A comparison of the two block types will show whether $\mathrm{C}-\mathrm{R}$ compatibility affects the controlled Stroop-like effects.

\section{Method}

Subjects. Seven female and 3 male students from the University of California participated in the experiment for course credit. Their mean age was 21 years (ranging from 19 to 25 years). They were all right-handed as assessed by a questionnaire (Coren, Porac, \& Duncan, 1979) and had normal or corrected-to-normal vision.

Apparatus and Stimuli. The stimuli were presented on an IBM AT personal computer in a darkened room. The stimuli were dark blue letters on a light gray background. The English words ABOVE and BELOW were shown above or below an asterisk. The entire word-asterisk configuration appeared in either the right or the left VF.

A plus sign (" +") subtending $0.48^{\circ}$ served as a central fixation point. The asterisk appeared $3.57^{\circ}$ of visual angle to the right or left of this central fixation point. The words appeared $1.06^{\circ}$ above or below the asterisk and subtended $2.44^{\circ}$ horizontally and $0.73^{\circ}$ vertically. The closest edge of any word appeared $2.27^{\circ}$ away from the center. A viewing hood ensured a constant viewing distance of $70.5 \mathrm{~cm}$.

Conditions. Five different conditions were presented, each in a block of 120 trials. The first three conditions were identical for both VFs. In Condition 1 ( $50 \%$ condition), $50 \%$ of the trials were congruent (ABOVE appearing above and BELOW appearing below the cross) and 50\% were incongruent (ABOVE appearing below and BELOW appearing above the cross). In Condition 2 ( $80 \%$ condition), there were $80 \%$ congruent and $20 \%$ incongruent trials in both VFs. In Condition 3 ( $20 \%$ condition), $20 \%$ of the trials were congruent and $80 \%$ were incongruent in both VFs (Conditions 2 and 3 represented the homogeneous blocks). In the last two conditions, the probabilities of congruent and incongruent trials were reversed between VFs (mixed blocks). In Condition 4, $80 \%$ of the trials were congruent and $20 \%$ were incongruent in the left VF (LVF), whereas 
$20 \%$ were congruent and $80 \%$ were incongruent in the right VF (RVF). In Condition 5, these probabilities were reversed: $20 \%$ of the trials were congruent and $80 \%$ were incongruent in the LVF and $80 \%$ were congruent and $20 \%$ were incongruent in the RVF. For each condition, the order of trials was randomized for each subject, with the constraint that the two words and VFs were completely balanced within subjects. The order of conditions was counterbalanced across subjects according to sequentially balanced Latin squares, such that each condition was followed twice by every other condition and each condition was given twice at each sequential position.

Procedure. The subjects were instructed to respond to the identity of the words and not to their spatial position (see Logan \& Zbrodoff, 1979). The different conditions were explained to them, and they were encouraged to use their knowledge of where each word was likely to appear to facilitate their responses. Before each block of trials, a message on the screen indicated the probability with which each word would appear in each position on the screen, and advised the subjects that they could start the trials by pressing any key. The subjects were told to respond as quickly as possible without making errors. They were instructed to keep their eyes fixated at the center of the screen and to rest their heads against the viewing hood.

Each trial began with a central fixation sign (" + ") for $500 \mathrm{msec}$. followed by a blank interval of $500 \mathrm{msec}$, after which the fixation dot reappeared together with the word-asterisk configuration for $100 \mathrm{msec}$. The next trial began after the subject had given a response, with an intertrial interval of at least $2 \mathrm{sec}$. Half of the subjects responded with the right hand and the other half responded with the left hand. The index finger was used to respond to the word ABOVE and the middle finger was used to respond to the word BELow. The response keys were centered with respect to the display. The first five trials after each break were discarded. In addition, RTs longer than $1.5 \mathrm{sec}$ were discarded $(0.3 \%)$

\section{Results and Discussion}

The mean error rate was low $(3.0 \%)$ and was not statistically analyzed.

$\mathbf{5 0 \%}$ condition. RTs in the $50 \%$ condition are given in Table 1. Responses were faster to congruent trials than to incongruent trials (the typical Stroop effect). A $2 \times$ 2 [VF $\times$ congruency (congruent/incongruent trials)] repeated measures analysis of variance (ANOVA) showed a main effect of congruency $\left[F(1,9)=7.26, M S_{\mathrm{e}}=\right.$ $579.0, p<.03$ ]. No effects with VF approached significance (both $F_{\mathrm{s}}<1$ ).

$\mathbf{8 0 \%} / \mathbf{2 0 \%}$ conditions. Figure 1 displays the mean RTs across subjects for conditions with $80 \%$ and $20 \%$ of congruent trials as a function of block type. Three important results are evident from Figure 1. First, there were

Table 1

Mean Correct Reaction Times (in milliseconds) in the $50 \%$ Conditions in Experiments 1, 3, and 4

\begin{tabular}{ccccccc}
\hline & \multicolumn{2}{c}{ LVF } & & \multicolumn{2}{c}{ RVF } \\
\cline { 3 - 4 } \cline { 5 - 6 } Experiment & C & I & & C & I \\
\hline 1 & & 451 & 472 & & 450 & 469 \\
3 & Session A & 580 & 596 & 546 & 563 \\
& Session B & 638 & 660 & 641 & 660 \\
& Session C & 643 & 676 & 608 & 627 \\
4 & & 712 & 714 & 706 & 731 \\
\hline
\end{tabular}

Note-C $=$ compatible trial types, $I=$ incompatible trial types.

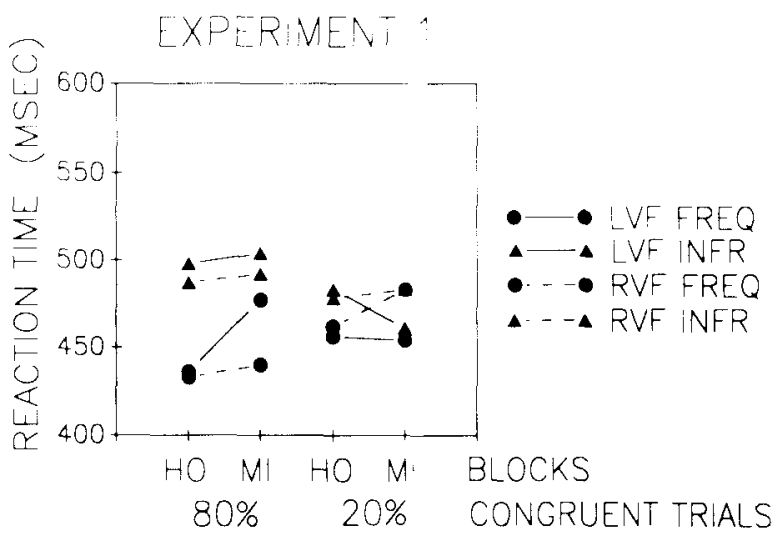

Figure 1. Mean correct reaction times in Experiment 1 as a function of block type and proportion of congruent trials. HO = homogeneous blocks, $\mathrm{MI}=$ mixed blocks, $80 \%=80 \%$ congruent trials, $20 \%=20 \%$ congruent trials, LVF $=$ target on the left side of the display, RVF = target on the right side of the display, FREQ $=$ frequent trial types, INFR = infrequent trial types.

pronounced controlled cuing effects. The subjects responded to frequent trial types faster than to infrequent ones. This means that when only $20 \%$ of the trials were congruent, the subjects responded to incongruent trials faster than to congruent ones, reversing the usual Stroop effect. Second, these controlled cuing effects were as pronounced in the homogeneous blocks as in the mixed blocks. Third, there were no VF differences.

A $2 \times 2 \times 2 \times 2$ [probability $(80 \% / 20 \%$ congruent trials) $\times \mathrm{VF} \times$ trial type (frequent/infrequent) $\times$ block type (homogeneous/mixed)] repeated measures ANOVA confirmed a main effect for trial type $[F(1,9)=69.42$, $\left.M S_{\mathrm{e}}=541.28, p<.001\right]$, which was modified by a significant interaction of probability $\times$ trial type $[F(1,9)=$ 9.64, $\left.M S_{\mathrm{e}}=1.359 .30, p<.02\right]$. Consistent with the literature (Logan, 1980; Logan \& Zbrodoff, 1979), this interaction indicates that controlled cuing effects were reduced when $20 \%$ of the trials were congruent compared to when $80 \%$ were congruent. The interaction of trial type $x$ block type, which would indicate a difference in controlled cuing effects between homogeneous and mixed blocks, was far from significant $(F<1.66, p>.10)$.

There were trends for the interactions of probability $x$ $\mathrm{VF}\left[F(1,9)=3.45, M S_{\mathrm{e}}=2,445.82, p<.10\right]$ and probability $\times \mathrm{VF} \times$ block type $\left[F(1,9)=4.11, M S_{\mathrm{e}}=\right.$ $1,097.58, p<.08]$. These interactions indicate that RTs tended to be faster when $80 \%$ of the trials were congruent in the RVF and $20 \%$ in the LVF than in the opposite arrangement. This result suggests that some stimulus features (e.g., the right-top position) and some response features (e.g., index finger) may be more salient than others (e.g., right-bottom position, middle finger) and may therefore produce faster overall response decisions.

In summary, the controlled Stroop-like effects demonstrated by Logan and associates (Logan, 1980; Logan \& Zbrodoff, 1979) were replicated in this task with VF presentation. Consistent with the findings of Logan and 
associates, controlled cuing effects were reduced when only $20 \%$ of the trials were congruent.

There was no difference in cuing efficiency between homogeneous and mixed blocks. As mentioned before, corresponding precues (e.g., the upper location in each VF) were associated with the same response finger in the homogeneous blocks, whereas the corresponding precues in the mixed blocks were associated with different response fingers. Therefore, precue-response compatibility was lower in the mixed than in the homogeneous block types. It is possible that in order for compatibility to affect cuing efficiency, a cue must be associated with more than one response alternative. This will be shown to be the case in Experiments 3 and 4, which included four response alternatives.

\section{EXPERIMENT 2 MEMORY LOAD}

Experiment 1 showed that controlled cuing effects were as pronounced in the mixed blocks with reduced $C-R$ compatibility as in the homogeneous blocks. In Experiment 2, we examined whether the resources involved in controlled cuing effects are general enough to be affected by a central capacity load. Subjects were asked to retain four letters in memory on each trial. Four letters are sufficient to produce interference effects when the interval between the onset of the first letter to be memorized and the target stimulus is relatively short (less than $3 \mathrm{sec}$ ), and if both tasks are presented visually (e.g., see Chow \& Murdock, 1975, 1976).

\section{Method}

Subjects. Four female and 4 male students at the University of California participated for course credit. Their mean age was 20 years (ranging from 18 to 22 years). They were all right-handed (Coren et al., 1979) and had normal or corrected-to-normal vision. None of the subjects had participated in Experiment 1. Three of the subjects were replacements for subjects whose data could not be used because their accuracy rate on reporting the four letters was below $80 \%$ correct (all other subjects had greater than $90 \%$ correct on the letters).

Apparatus and Procedure. The same apparatus as in Experiment 1 was used. Four blocks of 60 trials each were run with $80 \%$ (or $20 \%$ ) congruent trials in both VFs (homogeneous blocks) or with reversed probabilities in each VF (mixed blocks). To keep the experiment short enough to be completed within a 1-h session, the $50 \%$ condition was not included. On each trial, a random sequence of four letters, chosen randomly from the set $q, w, r, t, y, p, g$, $\mathrm{f}, \mathrm{l}, \mathrm{s}$, was presented for $1.5 \mathrm{sec}$, followed by the central fixation cross for $1 \mathrm{sec}$. After a blank interval of $800 \mathrm{msec}$, the word-x configuration appeared for $100 \mathrm{msec}$

The subjects used the computer keyboard to respond. They were instructed first to indicate the identity of the words as fast as possible, and then to type in the four letters that they had seen in the correct sequence. After typing in the four letters, they immediately put their index and middle fingers back on the two response keys in the middle of the keyboard for the words ABOVE and BELOW. The next trial began $1 \mathrm{sec}$ after they had typed in the last of the four letters. A $400-\mathrm{Hz}$ tone sounded for $500 \mathrm{msec}$ if an incorrect response to the letters was given. Half of the subjects used the right hand for responding and the other half used the left hand. The first five trials of each block were discarded as practice trials.

\section{Results and Discussion}

Only trials on which the four letters retained in memory were correctly reported in their correct sequence were included in the analysis ( $94 \%$ of all trials). For the main task, RTs longer than $1.5 \mathrm{sec}$ were discarded $(0.7 \%)$. The overall error rate was too low $(0.94 \%)$ to be analyzed statistically. The mean RTs are given in Figure 2. The figure shows that cuing effects were reduced in the mixed blocks, especially for stimuli presented on the left.

A $2 \times 2 \times 2 \times 2$ [probability $\times$ VF $\times$ trial type (frequent/infrequent) $\times$ block type] repeated measures ANOVA confirmed a significant main effect of trial type $\left[F(1,7)=29.05, M S_{\mathrm{e}}=762.73, p<.01\right]$ and an interaction of VF $\times$ trial type $\times$ block type $[F(1,7)=5.70$, $\left.M S_{\mathrm{e}}=1,105.25, p<.05\right]$. No other effects in the analysis approached significance. The interaction of trial type $\times$ block type was far from reliable $(F<1)$. The analysis confirms that controlled cuing effects were reduced in the mixed blocks for targets on the left, but not for those on the right.

The memory load of four letters was introduced to compete for attentional resources. Its overall effect was relatively weak, which fits well with evidence that controlled cuing effects in this manual response task rely heavily on motor-specific resources (Logan, Zbrodoff, \& Fostey, 1983). The memory load interfered with controlled cuing effects in the mixed relative to the homogeneous blocks for targets on the left, but not for targets on the right. In other words, the four-letter memory load apparently produced a bias to concentrate resources on response-decision rules for targets on the right and withdraw resources from targets on the left.

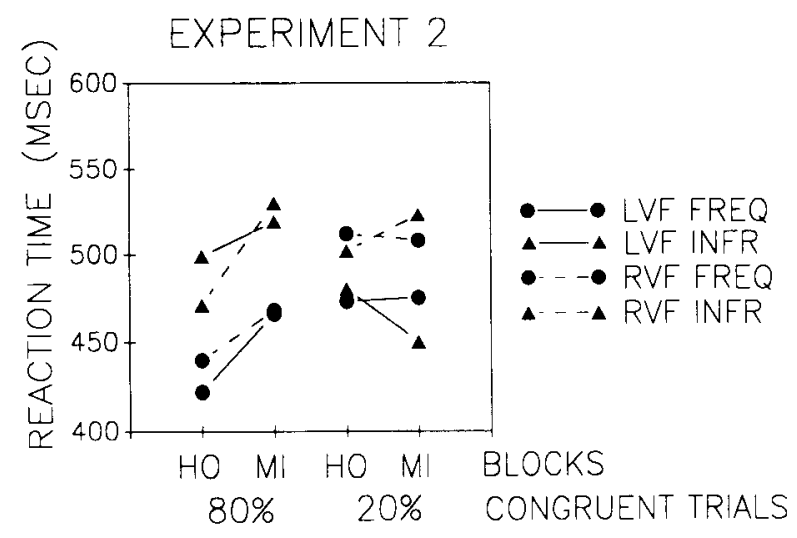

Figure 2. Mean correct reaction times in Experiment 2 as a function of block type and proportion of congruent trials. $\mathrm{HO}=$ homogeneous blocks, $\mathrm{MI}=$ mixed blocks, $80 \%=80 \%$ congruent trials, $20 \%=20 \%$ congruent trials, LVF $=$ target on the left side of the display, RVF = target on the right side of the display, FREQ = frequent trial types, INFR = infrequent trial types. 


\section{EXPERIMENT 3 FOUR RESPONSE ALTERNATIVES: HEMISPHERE-SPECIFIC RESOURCES}

In the first two experiments, two response alternatives had been used, with each finger being associated with two stimulus positions on the screen. In the third experiment, subjects responded with four response fingers (two on each hand). Increasing the number of response alternatives increases the complexity of response decisions and slows RTs (e.g., see Teichner \& Krebs, 1974). Therefore, as in situations with low compatibility, reduced controlled cuing effects were expected, relative to when there were only two response alternatives.

In addition, in Experiment 3, we examined whether separate hemispheric cognitive systems are involved when response-selection decisions for each hand are made. If there are mutually inaccessible, specific resource supplies in each hemisphere (Friedman \& Polson, 1981; Friedman, Polson, \& Dafoe, 1988; Friedman, Polson, Dafoe, $\&$ Gaskill, 1982), then independent access to these pools by lateralization of the task to one or the other hemisphere should minimize cross-talk between the two systems. There should be minimal interference between the response-selection rules for each hand, and the controlled cuing effects should be maximal with complete lateralization of the task. Since automatic cuing effects should not depend on any resources, complete lateralization of the task was not expected to affect cuing efficiency in the $50 \%$ condition.

Experiment 3 was run in three sessions. In all sessions, both hands were used for responding. Corresponding fingers on each hand were associated with the same response alternative (e.g., the index finger on each hand was used to respond "above"). In the homogeneous blocks, corresponding fingers were associated with corresponding positions on the screen (e.g., the index fingers with the response "above" to stimuli in the upper position). In the mixed blocks, corresponding fingers on each hand were associated with different positions on the screen (e.g., the index finger of the left hand was used for the response "above" to stimuli in the upper position in one VF, whereas the index finger of the right hand was used for the response "below" to stimuli in the lower position in the other VF).

\section{Method \\ Subjects. Four female and 6 male volunteers with a mean age of 28 years (ranging from 22 to 33 years) served as subjects for three 1 -h sessions. They were all right-handed as assessed by a ques- tionnaire (Oldfield, 1971, translated into German) and had normal or corrected-to-normal vision. Four of the subjects who did not be- long to the staff of the Institute of Behavioral Sciences, SFIT Zurich, were paid sFr. 30 for their participation. None of the subjects had participated in a previous experiment.}

Apparatus and Stimuli. The stimuli were shown on a SIRIUS-1 personal computer in a darkened room. The stimuli were dark letters on a light green background (reverse video). The stimuli were the German words OBEN (above) and UNTEN (below), written in capital letters and appearing above or below a lowercase $x$ in the right or left VF. Each letter subtended a visual angle of $0.40^{\circ}$ horizontally and $0.81^{\circ}$ vertically. The cross subtended $0.54^{\circ}$ horizontally $\times 0.36^{\circ}$ vertically. The word OBEN subtended $1.88^{\circ}$ horizontally, and the word UNTEN subtended $2.42^{\circ}$. The closest edge of any word appeared $3.04^{\circ}$ to the right or left of a central fixation point and was centered $1.34^{\circ}$ above or below the cross, which appeared laterally $4.29^{\circ}$ from the fixation point. A headrest with a viewing hood ensured a constant distance of $64 \mathrm{~cm}$ from the screen.

Sessions. Each subject completed three separate sessions at weekly intervals. The same four response keys were used in all three sessions. In Session A, the subjects sat upright in front of the screen. They responded with the right hand on the right-hand keys to stimuli in the RVF and with the left hand on the left-hand keys to stimuli in the LVF. Thus, stimulus input and response initiation were completely lateralized to a single hemisphere.

In Session B, the subjects responded with their hands crossed. The right hand was on the left-side keys and responded to stimuli in the LVF, whereas the left hand was on the right-hand keys and responded to stimuli in the RVF. The motor programs for responses to stimuli on the left, which are projected to the right hemisphere, thus involved the right hand (left hemisphere), and vice versa. Thus, the translation of response codes to motor commands involved a transfer between hemispheres.

In Session C, the subjects tilted their heads by $90^{\circ}$. The words now appeared (rotated by $90^{\circ}$ ) in their upper and lower central visual fields. The headrest was also rotated by $90^{\circ}$ and was adjusted so that the fixation dot was still in the center of the field of vision. The subjects used a reclining chair positioned sideways and supporting cushions. They sat as upright as possible, such that the head fit the viewing hood but the position was not too uncomfortable. As in Session A, they responded with the right hand on the righthand keys to stimuli on the right and with the left hand on the lefthand keys to stimuli on the left. In this arrangement, the stimuli were projected to both hemispheres. The subjects had strong environmental cues (upright body position and visibility of the experimental set-up), and their performance was expected to reflect the environmental rather than the retinal frame of reference (see Corballis, Anuza, \& Blake, 1978; Ladavas \& Moscovitch, 1984).

Thus, the stimuli appeared in the right and left (Sessions $A$ and $B$ ) or the central VF (Session C). They were presented to the same (Session A) or the opposite (Session B) hemisphere that initiated the response, or to both hemispheres (Session C). Head tilt rather than central presentation was chosen in Session $C$ so that spatial $S-R$ compatibility was similar in all sessions. Throughout Experiment 3, the right-side keys were used to respond to stimuli on the right, and the left-side keys were used to respond to stimuli on the left.

Each of the six possible orders of sessions was used at least once but never more than twice. In Session C, half of the subjects tilted their heads to the right, the other half to the left. Half of the subjects used the index finger on each hand to respond to the word OBEN and the middle finger of each hand to respond to the word UNTEN; the other half did the opposite. At least 2, but never more than 3 , subjects were assigned to each of the four possible headtilt/response-key combinations.

Conditions and Procedure. The conditions and procedure were the same as in Experiment 1, with the following exceptions. Due to system constraints, the stimuli were slightly more peripheral than in the previous experiments. Therefore, exposure time was increased by $50-150 \mathrm{msec}$ to roughly equate the visibility of the stimuli to the previous experiments. The first 20 trials of the first block and the first 10 trials of all subsequent blocks were considered practice trials. The subjects were given short breaks after each block of 120 
trials. They were allowed to withhold a response (and thus interrupt the presentation sequence) if necessary.

\section{Results and Discussion}

An upper limit of $1.5 \mathrm{sec}$ was imposed on RTs, and slower responses were discarded (1.3\%). Responses to the wrong half-field $(0.2 \%)$ were also discarded.

$\mathbf{5 0 \%}$ condition. The mean correct RTs for each session are given in Table 1 . A $3 \times 2 \times 2$ [session (normal, crossed hands, tilted head) $\times \mathrm{VF} \times$ congruency] repeated measures ANOVA was performed on the mean correct RTs. RTs were faster in Session A $(571 \mathrm{msec})$ than in the other sessions $[\mathrm{B}, 650 \mathrm{msec}$;, $638 \mathrm{msec}$; $\left.F(2,18)=5.12, M S_{\mathrm{e}}=13,997.43, p<.02\right]$. This result is due to the fact that crossing the hands (as in Session B) prolongs RTs due to a mismatch of the location of the response (e.g., the keys on the left) and the anatomical location of response production (e.g., the right hand; Nicoletti, Umiltà, \& Ladavas, 1984), and is also found when only the response goals (e.g., the response keys) but not the response effectors are crossed (Riggio, Gawryszewski, \& Umiltà, 1986). In addition, in Session $\mathrm{C}$, the words were read under head tilt so that the letters appeared rotated by $90^{\circ}$, making them harder to identify.

RTs were faster to stimuli on the right than to those on the left $\left[F(1,9)=7.16, M S_{e}=2,486.72, p<.03\right]$. However, a significant session $\times$ VF interaction $[F(2,18)$ $\left.=4.06, M S_{\mathrm{e}}=1,292.09, p<.04\right]$ indicated that this relation held only in the normal and tilted-head sessions. RTs were faster to congruent than to incongruent trial types $\left[F(1,9)=6.36, M S_{\mathrm{e}}=2,080.46, p<.03\right]$, reflecting the typical automatic Stroop-like effect. None of the interactions with trial type approached significance (both $F \mathrm{~s}<1$ ). Therefore, none of the task manipulations had any effect on the automatic processing of the cuing dimension.

$\mathbf{8 0} \% / 20 \%$ conditions. Figure 3 shows the mean correct RTs for each session. The figure demonstrates that the controlled cuing effects reported by Logan and Zbrodoff (1979) and Logan (1980) were replicated again with four response alternatives. The figure also shows that these controlled cuing effects were reduced in the mixed relative to the homogeneous blocks in all three sessions. As in the $50 \%$ condition, RTs in Session A were faster than in the other two sessions.

A $3 \times 2 \times 2 \times 2 \times 2$ [session $\times$ probability $\times \mathrm{VF}$ $\times$ trial type (frequent/infrequent) $\times$ block type] repeated measures ANOVA confirmed a main effect for session $\left\{F(2,18)=7.48, M S_{\mathrm{e}}=75,501.10, p<.01\right]$. No other interactions with session approached significance [all $F \mathrm{~s}(2,18)<2.59, p \mathrm{~s}>.10]$.

The main effect for trial type was also significant $\left[F(1,9)=37.35, M S_{\mathrm{e}}=2,211.32, p<.001\right]$. This effect was modified by interactions of probability $\times$ trial type $\left[F(1,9)=30.87, M S_{\mathrm{e}}=2,077.28, p<.001\right]$, probability $\times$ VF $\left[F(1,9)=3.72, M S_{\mathrm{e}}=5,200.04, p<\right.$ $.09]$, trial type $\times$ block type $\left[F(1,9)=11.77, M S_{\mathrm{e}}=\right.$ $1,705.43, p<.01]$, probability $\times$ trial type $\times$ block type
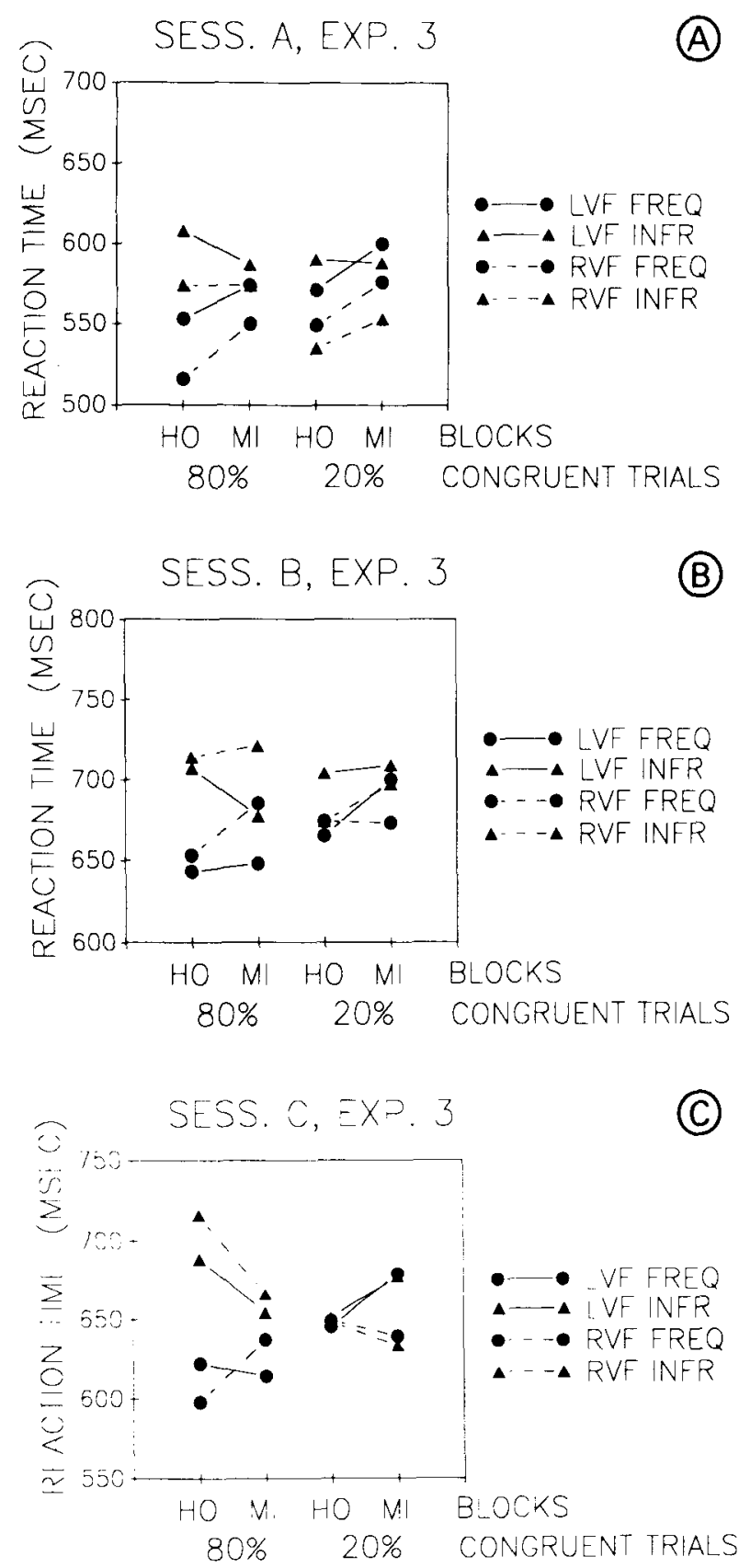

Figure 3. (A) Mean correct reaction times in Session A of Experiment 3 as a function of block type and proportion of congruent trials. (B) Mean correct reaction times in Session B of Experiment 3 as a function of block type and proportion of congruent trials. $(C)$ Mean correct reaction times in Session $\mathbf{C}$ of Experiment 3 as a function of block type and proportion of congruent trials. HO = homogeneous blocks, $\mathrm{MI}=$ mixed blocks, $80 \%=80 \%$ congruent trials, $20 \%=$ $20 \%$ congruent trials, LVF = target on the left side of the display, RVF = target on the right side of the display, FREQ = frequent trial types, INFR = infrequent trial types.

$\left[F(1,9)=6.11, M S_{\mathrm{e}}=1,305.51, p<.04\right]$, and probability $\times$ VF $\times$ trial type $\times$ block type $[F(1,9)=15.52$, $\left.M S_{\mathrm{e}}=196.49, p<.01\right]$. In other words, the controlled cuing effects were pronounced in the homogeneous blocks, but were significantly reduced in the mixed blocks. 
This reduction was more pronounced when $80 \%$ of the trials were congruent than when only $20 \%$ were congruent. In the RVF, controlled cuing effects were abolished when only $20 \%$ of the trials were congruent in either block type. No other effects approached significance [all $F_{\mathrm{s}}(1,9)<3.28$, ps $>.10$ ].

The mean error rates were below $5 \%$ in all conditions (1.32\% in Session A, $1.61 \%$ in Session B, and 3.64\% in Session C). These error rates were considered too low for a meaningful analysis.

Comparison of Session A and Experiment 1. The above ANOVAs with experiment as an additional between-subjects factor were performed to compare Session A of Experiment 3, in which there were four response alternatives, to Experiment 1, which had two response alternatives. For the $50 \%$ condition, the interaction of VF $\times$ experiment $\left[F(1,18)=5.99, M S_{\mathrm{e}}=\right.$ $799.46, p<.03$ ] confirmed the fact that, in contrast to Session A of Experiment 3, no RVF advantage was present in Experiment 1 with two response alternatives.

The analysis for the $80 \% / 20 \%$ conditions confirmed the interaction of VF $\times$ experiment $\left[F(1,18)=4.36, M S_{\mathrm{e}}=\right.$ $3,900.43, p<.06]$. The interaction of trial type $\times$ experiment $\left[F(1,18)=8.37, M S_{\mathrm{e}}=593.09, p<.01\right]$ reflected the fact that the overall controlled cuing effects were strongly reduced in Session A of Experiment 3 (15 msec), as compared to Experiment 1 ( $31 \mathrm{msec})$.

Summary. The major results of Experiment 3 can be summarized by three points. First, responding with two hands in uncrossed positions, as in Session A, introduced a consistent RVF advantage as compared to Experiment 1 (with only a single response hand). The fact that lateralized stimulus presentation was not sufficient by itself to produce this RVF advantage suggests that it is due to the correspondence of stimulus presentation side, response side, and response hand.

Second, the controlled Stroop-like effects were significantly reduced in the mixed relative to the homogeneous blocks. As mentioned earlier, the corresponding fingers of each hand were associated with corresponding stimulus positions in the homogeneous blocks, but not in the mixed blocks. In other words, precuing effects were enhanced when a salient stimulus feature (e.g., upper position) could be mapped onto a salient response feature (e.g., index finger or inner key). The results show that C-R compatibility affects controlled Stroop-like effects in the same way that it affects precuing effectiveness in precuing tasks (e.g., see Hendrikx, 1986; Proctor \& Reeve, 1985; Reeve \& Proctor, 1984). This result suggests that, similar to other precuing effects, these controlled Stroop-like effects involve the response-decision stage.

Third, the present results demonstrate that hemispherespecific resources are not important for these responsedecision processes. No advantage obtained in Session A in either block type when stimulus encoding, response decision, and motor programming were neuroanatomically separated to each hemisphere. Therefore, hemisphere- specific resources do not seem to support the responsedecision processes that produce the controlled cuing effects, and no benefit accrued from access to mutually inaccessible hemisphere-specific resource supplies. This result is consistent with results from precuing studies, which show more efficient cuing effects for spatially compatible responses than for responses on the same hand (Proctor \& Reeve, 1985).

\section{EXPERIMENT 4 SPATIAL COMPATIBILITY}

Spatial compatibility is frequently used in precuing paradigms as a task manipulation that affects response decisions. In Experiment 4, the influence of spatial compatibility on controlled cuing effects was examined. Subjects responded with the right hand on the right-side keys to stimuli in the LVF and with the left hand on the leftside keys to stimuli in the RVF. With this manipulation, both $\mathrm{C}-\mathrm{R}$ and $\mathrm{S}-\mathrm{R}$ compatibility are reduced. As compared to Session A of Experiment 3, reduced controlled cuing effects were expected due to the reduced spatial $C-R$ compatibility.

The previous experiment suggested that the correspondence of stimulus presentation side, response side, and response hand may be critical for an RVF advantage in RTs. Experiment 4 will show whether the correspondence of response side and response hand alone is sufficient to produce this RVF advantage.

\section{Method}

Subjects. One female and 9 male volunteers with a mean age of 28.3 years (ranging from 20 to 35 years) served as subjects. They were all right-handed (Oldfield, 1971) and had normal or correctedto-normal vision. None of the subjects had participated in any previous experiment.

Apparatus, Stimuli, and Procedure. The experimental procedure was identical to that of Session A of Experiment 3, except that the subjects were instructed to respond with the right hand on the right-side keys to stimuli in the LVF and with the left hand on the left-side keys to stimuli in the RVF.

\section{Results and Discussion}

For each subject, RTs greater than $1.5 \sec (4.98 \%)$ and responses to the wrong side of the display $(0.33 \%)$ were discarded.

50\% condition. The mean correct RTs given in Table 1 show an automatic Stroop-like effect (14 msec). However, in a $2 \times 2$ (VF $\times$ congruency) repeated measures ANOVA, no effects approached significance (all $F \mathrm{~s}<1$ ).

80\%/20\% conditions. The mean RTs across subjects are shown in Figure 4 . The figure shows pronounced controlled cuing effects. In most conditions, frequent trial types resulted in faster RTs than did infrequent ones. In the mixed blocks, controlled cuing effects were reduced for RVF stimuli (responded to on the left), but not for LVF stimuli (responded to on the right).

A comparison of the homogeneous and mixed blocks by a $2 \times 2 \times 2 \times 2$ (probability $\times \mathrm{VF} \times$ trial type $\times$ 


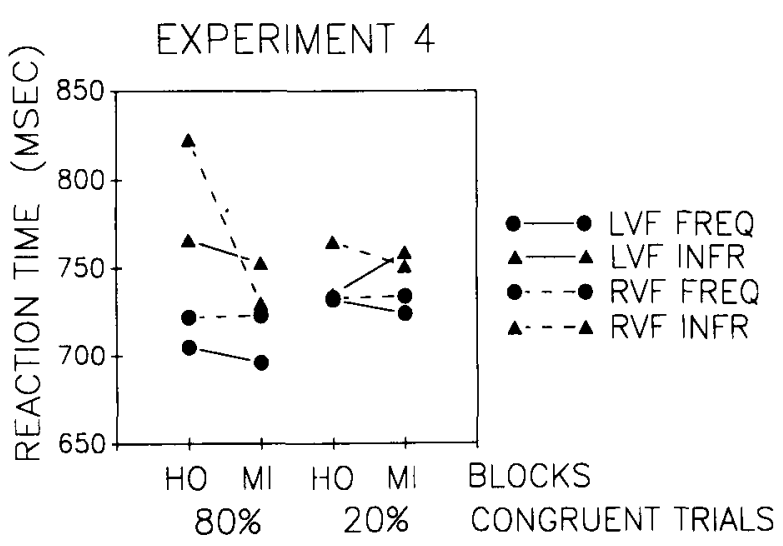

Figure 4. Mean correct reaction times in Experiment 4 as a function of block type and proportion of congruent trials. $\mathrm{HO}=$ homogeneous blocks, $\mathrm{MI}=$ mixed blocks, $80 \%=80 \%$ congruent trials, $20 \%=20 \%$ congruent trials, $L V F=$ target on the left side of the display, RVF = target on the right side of the display, FREQ $=$ frequent trial types, INFR = infrequent trial types.

block type) repeated measures ANOVA confirmed a significant main effect of trial type $\left[F(1,9)=20.36, M S_{\mathrm{e}}=\right.$ $3,041.93, p<.01]$ and a trend in the interaction of VF $\times$ trial type $\times$ block type $\left[F(1,9)=3.59, M S_{\mathrm{e}}=\right.$ $3,198.71, p<.10]$. The interaction of trial type $\times$ block type was not reliable $[F(1,9)<1.65, p>.10]$. The mean error rate $(3.3 \%)$ was considered too low for statistical analysis.

Comparison of Experiment 3 and Experiment 4. Inspection of Figures $3 \mathrm{~A}$ and 4 shows that the response pattern for LVF stimuli (responded to on the left) in Session A of Experiment 3 resembles that for RVF stimuli (responded to on the left) in Experiment 4, and vice versa. In other words, the response pattern seems to be determined by the response side rather than by the presentation side of the stimuli. The same ANOVAs as above were run with experiment (Session A of Experiment 3 vs. Experiment 4) as an additional between-subjects factor.

For the $50 \%$ condition, a significant interaction of VF $\times$ experiment $[F(1,18)=10.50, p<.01]$ was apparent, but no effects for congruency emerged (all $F \mathrm{~s}<1$ ). Therefore, variations in spatial S-R compatibility did not affect the automatic processing of the position information.

For the $80 \% / 20 \%$ conditions, the ANOVA confirmed significant interactions of experiment $\times \operatorname{VF}[F(1,18)=$ 7.86, $\left.M S_{\mathrm{e}}=4,991.47, p<.05\right]$, experiment $\times$ block type $\times \operatorname{VF}\left[F(1,18)=3.60, M S_{\mathrm{e}}=2,004.36, p<.08\right]$, experiment $\times$ trial type $\left[F(1,18)=6.49, M S_{\mathrm{e}}=\right.$ $1,843.41, p<.05]$, and experiment $\times$ block type $\times$ $\mathrm{VF} \times$ trial type $\left[F(1,18)=4.65, M S_{\mathrm{e}}=1,843.98, p<\right.$ $.05]$. In other words, comparison of Experiment 4 with Session A of Experiment 3 demonstrates that in both experiments, controlled cuing effects in the mixed blocks were reduced for stimuli responded to on the left but not for those responded to on the right, independent of the stimulus presentation side. However, the overall RVF advantage in RTs was not present in Experiment 4.

In summary, with the $\mathrm{C}-\mathrm{R}$-incompatible arrangement of Experiment 4, controlled cuing effects in the mixed blocks tended to be reduced for stimuli responded to on the left. The response pattern across probabilities and block types to targets in each VF was reversed between Session A of Experiment 3 and Experiment 4. In Experiment 3 (see Figure 3), controlled cuing effects in the mixed blocks were more pronounced for RVF stimuli responded to on the right than for LVF stimuli responded to on the left. In Experiment 4 (see Figure 4), however, cuing effects in the mixed blocks were more pronounced for LVF stimuli responded to on the right than for RVF stimuli responded to on the left.

These findings suggest that the controlled cuing effects were primarily affected by response-decision processes rather than by perceptual processes. Furthermore, the results demonstrate a bias to attend to the positionresponse contingencies for right-side over left-side responses. Other evidence suggests a rightward tendency in orienting (see Roy, Reuter-Lorenz, Roy, Copland, \& Moscovitch, 1987, for a review), and a rightward bias of early selective attention when competing input from both visual fields is available (Eglin, 1987). The present results suggest that a rightward bias may also be present at later processing stages, when response-decision rules are extracted and utilized.

Experiment 4 also demonstrated that even though the stimulus presentation side is not important for the pattern of controlled cuing effects, it is critical for an overall RVF advantage in RTs to occur. With only the correspondence of response side and response hand, the RVF advantage found in Session A of Experiment 3 was abolished.

\section{GENERAL DISCUSSION}

Stroop-like effects have recently been shown to involve automatic as well as controlled processing of the cuing dimension (Logan, 1980; Logan \& Zbrodoff, 1979). In the present study, it is demonstrated that these controlled cuing effects in a Stroop-like task are affected by task manipulations that affect response decisions. Automatic Stroop-like effects, on the other hand, were invariant across tasks.

\section{Automatic Processing of the Cuing Dimension}

The automatic Stroop-like effects in the $50 \%$ condition were not affected by increasing the number of response alternatives from two to four, nor by decreasing spatial compatibility. All between-experiment comparisons were far from significant (all $F_{\mathrm{S}}<1$ ). Thus, the automatic processing of the cuing dimension seems to run to completion without being affected by response-decision factors.

When two response hands were used, uncrossed, there was an RT advantage for the right hand responding to tar- 
gets on the right over the left hand responding to targets on the left (Experiment 3, Session A). However, visual field (or response side) never interacted with any of the other task variables. This finding corroborates the notion that automatic effects are relatively invariant constants superimposed on other RT effects (see LaBerge, 1981. for a review).

\section{Strategic Processing of the Position Information}

The following manipulations were used to affect response decisions in the present task. In all four experiments, conditions with $20 \%$ (as compared to $80 \%$ ) congruent trials showed less C-R compatibility, because each cuing alternative had to be associated with an opposing response alternative (e.g., the upper stimulus position with the response "below"). In the mixed (as compared to the homogeneous) blocks, C-R compatibility was reduced because there was no salient stimulus feature that could be associated with a salient response feature. In the last two experiments, the number of response alternatives was increased to four. Finally, in the last experiment, spatial compatibility was decreased.

$C-R$ compatibility and response complexity affected the controlled cuing effects in the same way they affect other precuing effects. Therefore, it seems that the controlled cuing effects in both types of tasks reflect similar responseselection mechanisms.

The third experiment was designed to examine the effects of stimulus and response lateralization in a withinsubjects design. Even though incomplete lateralization of the task prolonged RTs, it did not decrease controlled cuing efficiency in either block type. Therefore, the resources required at the response-selection stage could not be accessed more efficiently in the completely lateralized version of the task and do not seem to be hemisphere-specific.

Converging evidence from Experiment 2 with a memory load and Experiment 4 with low spatial C-R compatibility indicate a bias favoring response-selection rules for stimuli and responses on the right over those on the left. When only one response-decision rule can be maintained adequately, a bias seems to exist to maintain the rule for targets presented/responded to on the right rather than that for targets presented/responded to on the left. Response side was more important in determining this bias than was stimulus presentation side.

In summary, the results show that response-selection rules based on the frequency of congruent and incongruent trial types were established very consistently across the different experiments. Task manipulations presumed to affect response-decision processes competed for the resources required to establish the response-selection rules. Controlled cuing effects were reduced with four as compared to two response alternatives, and with low $\mathrm{C}-\mathrm{R}$ compatibility in the mixed blocks and in the $20 \%$ conditions. Therefore, the cuing effects that obtain in Stroop-like tasks must depend on response-decision processes.
This conclusion is in accord with other evidence that shows that Stroop-like effects occur at a stage when evidence in favor of one response relative to other responses is built up (Dunbar \& MacLeod, 1984; Logan \& Zbrodoff, 1979). With a different experimental approach. the present study explicitly associates these responseactivation processes with the response-decision stage.

When resources were scarce, rather than maintaining two different response-selection rules inefficiently, one was supported at the expense of the other. A clear bias existed to dedicate resources to the response-mapping rule appropriate for targets presented/responded to on the right. This result extends the evidence for a rightward bias for early orienting or selective functions of attention (e.g., see Eglin, 1987; Roy et al., 1987) to later responsedecision processes.

\section{REFERENCES}

Chow, S. L. \& Murdock, B. B., JR. (1975). The effect of a subsidiary task on iconic memory. Memory \& Cognition, 3, 678-688.

Chow, S. L., \& Murdock, B. B., JR. (1976). Concurrent memory load and the rate of readout from iconic memory. Joumal of Experimental Psychology: Human Perception \& Performance, 2, 179-190.

Corballis, M. C., Anuza, T., \& Blake, L. (1978). Tachistoscopic perception under head tilt. Perception \& Psychophysics, 24, 274-284.

COREN, S. PORAC, C., \& Duncan, J. (1979). A behaviorally validated self-report inventory to assess four types of lateral preference. Journal of Clinical Neuropsychology, 1, 55-64.

DUnbar, K., MACLEOD, C. M. (1984). A horse race of a different color: Stroop interference patterns with transformed words. Joumal of Experimental Psychology: Human Perception \& Performance, 10. 622-639.

EGLIN, M. (1987). Interference and priming within and across visual fields in a lexical decision task. Neuropsychologia, 25, 613-624.

Friedman, A., Polson, M. C. (1981). The hemispheres as independent resource-systems: Limited-capacity processing and cerebral specialization. Journal of Experimental Psychology: Human Perception \& Performance, 7, 1031-1058.

Friedman, A., Polson, M. C., Dafoe, C. G. (1988). Dividing attention between the hands and the head: Performance trade-offs between rapid finger tapping and vertal memory. Joumal of Experimental Psychology: Human Perception \& Performance, 14, 60-68.

Friedman, A., Polson, M. C., Dafoe, C. G., Gaskill, S. J. (1982) Dividing attention within and between hemispheres: Testing a multiple resources approach to limited-capacity information processing. Journal of Experimental Psychology: Human Perception \& Pefformance, 8, 625-650.

HendrJkX, A. J. P. (1986) Compatibility of precuing and of S-R mapping in choice reactions. Acta Psychologica, 62, 59-88.

LABERGE, D. (1981). Automatic information processing: A review. In J. Long \& A. Baddeley (Eds.), Attention and performance (Vol. 9, pp. 173-186). Hillsdale, NJ: Erlbaum.

Ladavas, E., Moscovitch, M. (1984). Must egocentric and environmental frames of reference be aligned to produce spatial S-R compatibility effects? Journal of Experimental Psychology: Human Perception \& Performance, 10, 205-215.

La Heis, W., Van der Heuden, A. H. C., Schreuder, R. (1985) Semantic priming and Stroop-like interference in word-naming tasks. Joumal of Experimental Psychology: Human Perception \& Performance, $11,62-80$

LoGAN, G. D. (1980). Attention and automaticity in Stroop and priming tasks: Theory and data. Cognitive Psychology, 12, 523-553.

LOGAN, G. D., \& ZBRodoff, N. J. (1979). When it helps to be misled: Facilitative effects of increasing the frequency of conflicting stimuli in a Stroop-like task. Memory \& Cognition, 7, 166-174. 
Logan, G. D., Zbrodoff, N. J., \& Fostey, A. R. W. (1983). Costs and benefits of strategy construction in a speeded discrimination task. Memory \& Cognition, 11, 485-493.

MCClain, L. (1983). Stimulus-response compatibility affects auditory Stroop interference. Perception \& Psychophysics, 33, 266-270.

MILLER, J. (1982). Discrete versus continuous stage models of human information processing: In search of partial output. Journal of $E x$ perimental Psychology: Human Perception \& Performance, 8, 273-296.

Miller, J. (1983). Can response preparation begin before stimulus recognition finishes? Joumal of Experimental Psychology: Human Perception \& Performance, 9, 161-182.

MilLER, J. (1985). A hand advantage in preparation of simple keypress responses: Reply to Reeve and Proctor (1984). Journal of Experimental Psychology: Human Perception \& Performance, 11, 221-233.

MILLER, J. (1987). Evidence of preliminary response preparation from a divided attention task. Journal of Experimental Psychology: Human Perception \& Performance, 13, 425-434.

Nicoletrı, R., Umiltà, C., \& Ladavas, E. (1984). Compatibility due to the coding of the relative position of the effectors. Acta Psychologica, 57, 133-143.

OLDFIELD, R. C. (1971). The assessment and analysis of handedness: The Edinburgh inventory. Neuropsychologia, 9, 97-113.

PosNer, M. I., \& SNyder, C. R. R. (1975). Facilitation and inhibition in the processing of signals. In P. M. A. Rabbitt \& S. Dornic (Eds.), Attention and performance (Vol. 5, pp. 669-682). New York: Academic Press.

Proctor, R. W., \& Reeve, T. G. (1985). Compatibility effects in the assignment of symbolic stimuli to discrete finger responses. Journal of Experimental Psychology: Human Perception \& Performance, 11 , 623-639.

Proctor, R. W., \& Reeve, T. G. (1986). A caution regarding use of the hint procedure to determine whether partial stimulus information activates responses. Perception \& Psychophysics, 40, 110-118.

Reeve, T. G., \& Proctor, R. W. (1984). On the advance preparation of discrete finger responses. Journal of Experimental Psychology: $\mathrm{Hu}$ man Perception \& Performance, 10, 541-553.

Reeve, T. G., \& Proctor, R. W. (1985). Nonmotoric translation processes in the preparation of discrete finger responses: A rebuttal of Miller's (1985) analysis. Joumal of Experimental Psychology: Human Perception \& Performance, 11, 234-241.

Riggio, L., GaWRYSZeWSKI, L. D. G, \& UMiltà, C. (1986). What is crossed in crossed-hand effects? Acta Psychologica, 62, 89-100.

Roy, E. A., Reuter-Lorenz, P., Roy, L. G., Copland, S., \& Moscovirch, M. (1987). Unilateral attention deficits and hemispheric asymmetries in the control of attention. In M. Jeannerod (Ed.), Neurophysiological and neuropsychological aspects of spatial neglect (pp. 25-39). Amsterdam: Elsevier.

Shiffrin, R. M., \& SCHNEIDER, W. (1977). Controlled and automatic human information processing: II. Perceptual learning, automatic attending, and a general theory. Psychological Review, 84, 127-190.

TeichNer, W. H., \& KREBS, M. J. (1974). Laws of visual choice reaction time. Psychological Review, 81, 75-98.

VIRZI, R. A., \& EGETH, H. E. (1985). Toward a translational model of Stroop interference. Memory \& Cognition, 13, 304-319.

(Manuscript received August 17, 1989; revision accepted for publication February 12, 1990.) 